\title{
VELIKHOV ELECTROTHERMAL INSTABILITY CANCELLATION BY A MODIFICATION OF ELECTRICAL CONDUCTIVITY VALUE IN A STREAMER BY MAGNETIC CONFINEMENT
}

\author{
JeAn P. Petit ${ }^{a}$, JeAn C. DORE ${ }^{b, *}$ \\ ${ }^{a}$ Research manager, Lambda Laboratory, France \\ $b$ Technical manager, Lambda Laboratory, France \\ * corresponding author: contact@lambda-laboratory.fr
}

\begin{abstract}
We present a method, confirmed experimentally, allowing the cancellation of Velikhov instability by operating a local magnetic field reduction along a lane, which enhances local electric conductivity and electron-gas collision frequency, due to a local passage into a Coulomb collision regime and the subsequent lowering of the Hall parameter below its critical value, close to 2 .
\end{abstract}

KEYWORDS: non equilibrium plasmas, MHD, Velikhov instability.

\section{INTRODUCTION}

Electrothermal instability was discovered by E. Velikhov, a student of Andrei Sakharov. His paper [20, presented at the international MHD congress in Newcastle in 1962, announced the complete ruin of the dreams of researchers and engineers working on electric current production processes by direct energy conversion of a fluid. The use of enthalpy associated with a gaseous supersonic flux obtained from hydrocarbon combustion was first considered. Theoretically, a $60 \%$ conversion efficiency was expected. However, problems were caused by the technological constraints due to wall and electrodes behaviour. Effectively, by adding a more easily ionizable substance to the fluid, caesium, its conductivity only allowed an acceptable output at temperatures above $3000{ }^{\circ} \mathrm{C}$. When this MHD energy production was seen to have failed, the Russians pushed the technology to extremes at the Kurtchatov Institute in Moscow with the U-25 generator. Its enormous MHD nozzle equipped with Zirconium oxyde electrodes only allowed a temperature of $2500^{\circ} \mathrm{C}$ to be attained. Given the non-linear increase of the ionization level according to the temperature, these few degrees of difference compared with the $3000{ }^{\circ} \mathrm{C}$ obliged the abandon of this formula despite the great amount of work done by several countries such as the USA, the UK, France, Germany, Italy, Poland, China and others. Another formula suggested by the American Kerrebrock 2, 3 consisted of aiming at a non-equilibrium operation with a considerably higher electronic temperature than that of the gas, i.e. of the heavy species, as is the case in a fluorescent tube. Then the MHD regime can be described by writing the energy conservation equation

$$
(\mathbf{V} \times \mathbf{B}) \cdot \mathbf{J}_{\mathrm{e}}=3 n_{\mathrm{e}} k\left(T_{\mathrm{e}}-T_{\mathrm{g}}\right) \sum_{s \neq \mathrm{e}} \frac{m_{\mathrm{e}}}{m_{s}} \delta_{s}\left\langle v_{\mathrm{e} s}\right\rangle,
$$

where $\delta_{s}$ refers to an ineslatic loss. This could not be applied to gases issuing from hydrocarbon combustion because of the presence of carbon dioxyde which hinders the electronic temperature increase by immediately absorbing the energy and maintaining a thermodynamic equilibrium. It can only concern heat carrying fluids, made up of rare gases seeded with caesium. This formula is seductive and makes use of electromotive fields with a high VB and involves high Hall parameter values. But in 1962 E. Velikhov predicted the appearance of the turbulence affecting the electron gas, making the plasma totally unhomogenous in a time period comparable to that of the establishment of the ionization itself. This theory was widely verified in laboratories.

\section{EXPERIMENTAL}

\subsection{EXPERIMENT IN HOT GAS}

In 1961 Bert Zauderer 21] used a shock tube as a short duration generator of hot swift rare gases flows, driven into a Faraday MHD converter, and got strong interaction parameters, due to the high electrical conductivity of Argon at $10000 \mathrm{~K}$ at one bar pressure (3000 Sievert/m). In 1967, using a similar installation, held in the Institute of Fluids Mechanics of Marseille, France, J. P. Petit managed [18, 17] to obtain the first and only non-equilibrium electric power production. The test gas was a mixture of helium and argon, where argon played the role of a seed. The formula only allowed experimentation for a maximum of $50 \mu$ s but was sufficient to allow a conclusive experiment. The idea was to make it that ionization development, leading the plasma to a Coulomb collision dominated regime, bring about a drop in the Hall parameter to below its critical value of around 2 in such a regime. This method allowed a stable operation to be attained, with a pressure of close to one bar, with a gas temperature of $4000^{\circ} \mathrm{C}$ and an electronic 


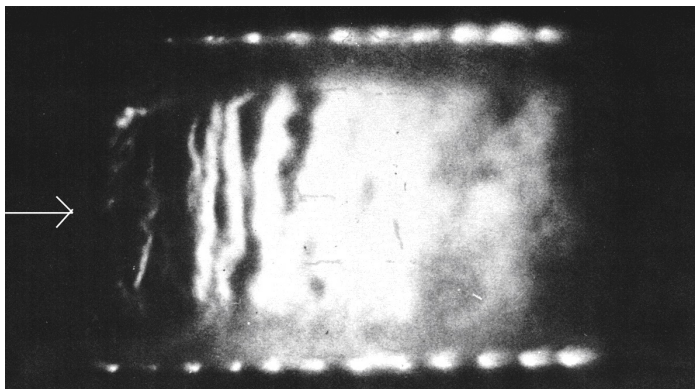

FiguRE 1. Velikhov instability, development and annihilation.

temperature up to $10000^{\circ} \mathrm{C}$. However calculations showed that it would not be possible to go below.

The image (Fig. 1) refers to an experiment managed in a schock driven wind tunnel, coupled to a Faraday MHD converter. Up and down a series of spots show the places of cathodes and anodes. The magnetic field, $2 \mathrm{~T}$, is perpendicular to the plane of the figure. The picture was taken in 1965, using a TRW electronicoptic fast image converter, the first one available in France. The arrow shows the inlet of the fluid. The constant section of the chanel is $5 \times 5 \mathrm{~cm}$ and the length of the MHD section is $10 \mathrm{~cm}$. We can see clearly on the image that instability begins to appear at the MHD channel's entry and is then absorbed by the ionisation development itself. This last rises the ion density. When the plasma is Coulomb dominated, the Hall parameter, whose value at the entrance is 8 , falls below the critical value. This was evoked in a previous paper [10]. This rapid rise of the collision frequency, due to large Coulomb cross section damps the Hall parameter, whose value, at the inlet, was close to 8 . If this passage to collision dominated regime is fast enough, it competes efficiently with the development of the electrothermal instability. In a collision dominated regime, in a two temperature plasma, the critical value of the Hall parameter is close to 2 . If $\beta$ is driven below this value, after the ionization is fully developed, the plasma becomes stable. See the right portion of the picture. Formulas giving the critical value of the Hall parameter and growth rate of the instability are given in 10 .

In our shock tube this Velikhov instability cancellation could not be managed down to $4000 \mathrm{~K}$. In a shock tube the temperature and the gas velocity are closely related. For lower temperatures, the induced electrical field $\mathbf{V} \times \mathbf{B}$ was not strong enough to give the required ionization velocity and too weak to drive the plasma into the Coulomb dominated plasma regime, so that this method was not technologically interesting.

\subsection{The IMPOSSIBILITY TO CONTINUE THIS RESEARCH.}

In France, at the end of the sixties, or, for other countries at the beginning of the seventies, the MHD electric power generation was progressively abandonned.
Budgets were completely cut, and the author, until retired, shifted to kinetic theory of gases, astrophysics and cosmology. After publishing some theoretical works about electrothermal instability [16] (see also [1]) he discovered in 1981 a second method, for Velikhov instability cancellation, firstly published at the French Academy of Science [8], then presented in the eight international MHD meeting, held in Moscow [6]. He supported this attendance by his own funding. Years passed. Due to the lack of funding J. P. Petit was unable to attend meetings in Tsukuba, Beijing, where his communications were accepted [12, 15] and finally gave up. The explanation of the rarity of publications about Velikhov instability cancellation is the following: this is a key problem for classified research, devoted to MHD controlled hypersonic flight in high altitude.

\subsection{BACK TO EXPERIMENTS IN COLD PLASMA}

He was retired since 5 years when young men suggested to restart private MHD research, out of any official institution. J. C. Doré had some place in his small garage. The team got funding, selling a book of J. P. Petit, and J. C. Doré built a small low pressure test installation. Then the work restarted. Thanks to this original funding system, the men of Lambda Laboratory could buy material and attend international meetings. The attention was concentrated on disk shaped accelerators systems, corresponding to previously published papers. The set of ideas can be found in the proceedings of reference [11. The final purpose is to show that shock waves and turbulence can be eliminated around diskshaped flying machines, by suitable Lorentz force field action [7, 15, 19, 12, 4, 13, 14, 15. The lack of other papers about this subject deserves the same explanation: in highly scientifically advanced countries (USA, Russia) all that stuff corresponds to highly classified research.

The successive experimental works achieved by J. C. Doré in his small garage are necessary steps towards this central goal. The Velikhov instability control is just one of them. In 2010 J. C. Doré and J. P. Petit presented a successful wall confinment experiment, close to a disk shaped machine by magnetic pressure gradient inversion [9]. Recently we achieved stable spiral current pattern, that will be the next presentation and publication. Back to the present subject the photo (Fig. 2) shows the extremely unhomogenous aspect, connected to the development of instability in low pressure air. The magnetic field, produced by a large coil, is perpendicular to the plane of the figure.

The photo (Fig. 2 made in Lambda Laboratory CCD camera F4 1s) shows the extremely unhomogenous aspect, connected to the development of instability. In the following (Fig. 3 made in Lambda Laboratory CCD camera F4 1s) we attenuated the magnetic field in a lane by reducing to 200 Gs. 


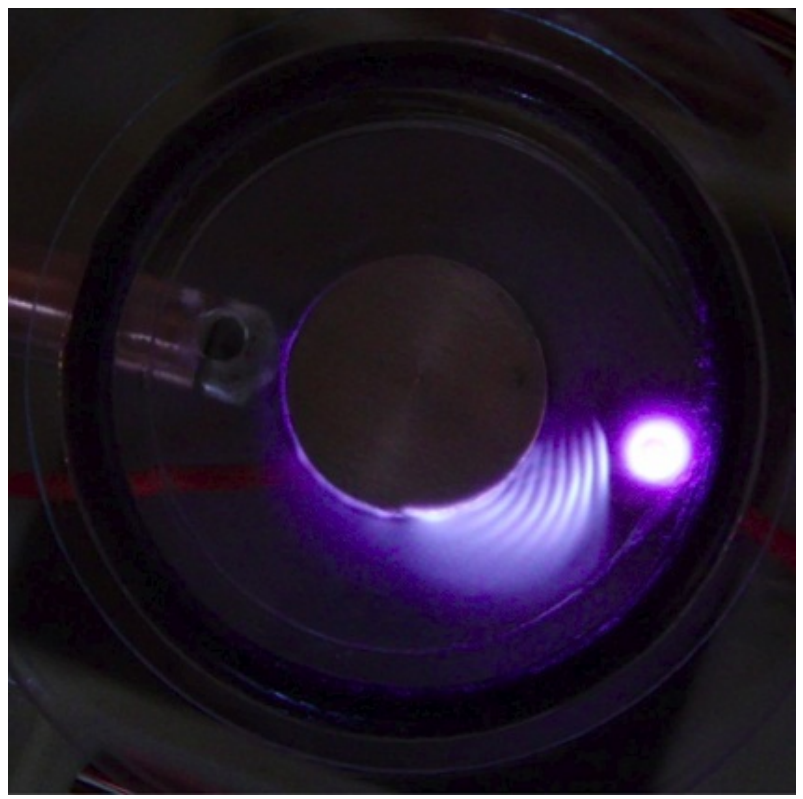

Figure 2. Instability.

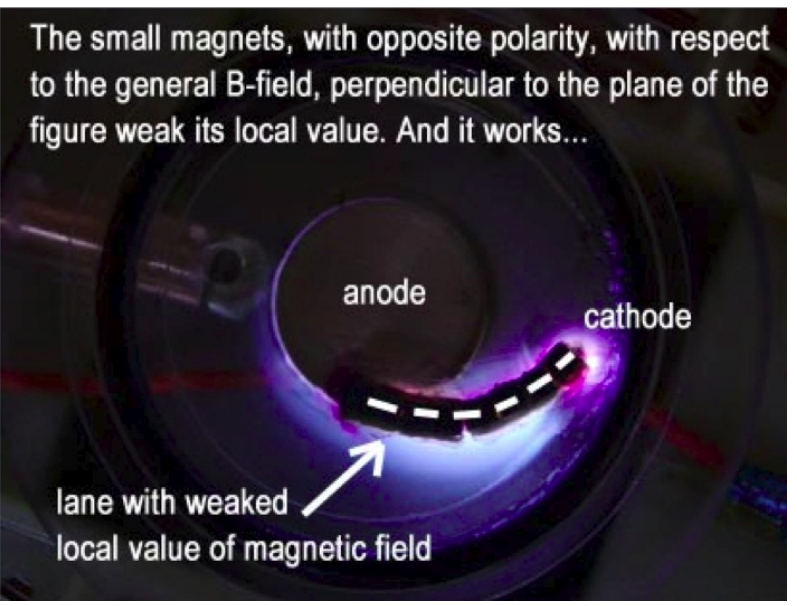

Figure 3. Controlled instability.

\section{Results And Discussion}

The current was thus incited to take the route of least electric resistance so as to bring the plasma, locally, into a Coulomb dominated regime, where we know that the Hall parameter critical value is then close to 2 . The local Hall parameter value becomes, locally, lower than this value, the plasma is then brought into a stable configuration. The experiment aims to create spiral currents in order to obtain a quasi radial force field, even though the local Hall parameter value is low. The discharge geometry is then completely controlled by the magnetic geometry and not by the Hall effect.

\section{Conclusion}

This electrothermal instability is one of the greatest problems of cold, non-equilibrium magnetized plasmas, particularly in relation to the development of equipment used at altitudes well above $30 \mathrm{~km}$, where the flight regime is necessarily hypersonic and where gas flow control towards the ramjets can be done using an MHD controlled inlet. Then Velikhov instability must then be totally cancelled [11. The intention of the Lambda Laboratory team is to extend the research to suck low density gas by Lorentz force action, around a disk shaped MHD aerodyne model. Beyond, we believe that strong sucking action on surrounding air can prevent the birth of shock waves and turbulence around a MHD aerodyne, moving in supersonic, and event hypersonic velocity in air. We think that silent and shockless flight is possible, at supersonic velocity, in dense air.

\section{ACKNOWLEDGEMENTS}

This research is solely sponsored by private funds. The authors thank all those who support us by private donations and without whom this research would not be possible. The authors thanks their collaborators, members of the Lambda Laboratory: Mathieu Ader, Xavier Lafont and for their technical help: Jacques Legalland, Jacques Juan, ...

\section{REFERENCES}

[1] M. G. Haines, A. H. Nelson. Analysis of the growth of electrothermal waves. In 10th symp. on Engineering Aspects of MHD. Cambridge, 1969.

[2] J. L. Kerrebrock. Non-equilibrium effects on conductivity and electrode heat transfer in ionized gases. Tech. Rep. technical note 4, AFOSR-165, Guggenheim Jet Propulsion Center, Caltech, Pasadena, California, 1960.

[3] J. L. Kerrebrock. Conduction in gases with elevated electron temperature. In Proc. 2nd Symp. Eng. Aspects of $M H D$, pp. 327-346. 1962.

[4] B. Lebrun. Approche theorique de la suppression des ondes de choc se formant autour d'un obstacle effile place dans un ecoulement supersonique d'argon ionise a l'aide de forces de Laplace (Theoretical study of shock wave annihilation around a flat wing in hot supersonic argon flow with Lorentz forces). Aix-Marseille University; Journal of Mechanics, France, 1987. Dir. by J. P. Petit. Engineer-Doctor thesis.

[5] J. P. Petit. Convertisseurs MHD d'un genre nouveau (New MHD converters). CRAS, French Academy of Sciences 281(11):157-160, 1975.

[6] J. P. Petit. Cancellation of the Velikhov instability by magnetic confinement. In 8th International Conference on the MHD Electrical Power Generation. Moscow, Russia, 1983.

[7] J. P. Petit. Is supersonic flight without shock wave possible? In 8th International Conference on MHD Electrical Power Generation. Moscow, Russia, 1983.

[8] J. P. Petit, M. Billiotte. Method for eliminating Velikhov instability. CRAS, Academy of Science 1981.

[9] J. P. Petit, J. C. Dore. Wall confinement technique by magnetic gradient inversion. In 3rd Euro-Asian Pulsed Power Conference (EAPPC2010). Jeju, Korea, 2010. (and in Acta Polonica A 121(3):611, March 2012). 
[10] J. P. Petit, J. Geffray. Non equilibrium plasma instabilities. In 2nd Euro-Asian Pulsed Power Conference (EAPPC2008). Vilnius, Lithuania, 2008. (and in Acta Physica Polonica A 115(6):1170-1173, June 2009).

[11] J. P. Petit, J. Geffray, F. David. MHD hypersonic flow control for aerospace applications. In 16th AIAA/DLR/DGLR International Space Planes and Hypersonic Systems and Technologies Conference (HyTASP). Bremen, Germany, 2009. AIAA-2009-7348.

[12] J. P. Petit, B. Lebrun. Shock wave cancellation in gas by Lorentz force action. In 9th International Conference on MHD Electrical Power Generation. Proceedings III, part 14.E - MHD flow, pp. 1359-1368. Tsukuba, Japan, 1986. Article 5.

[13] J. P. Petit, B. Lebrun. Shock wave annihilation by MHD action in supersonic flow. Quasi one dimensional steady analysis and thermal blockage. European Journal of Mechanics B/Fluids 8(2):163-178, 1989.

[14] J. P. Petit, B. Lebrun. Shock wave annihilation by MHD action in supersonic flows. Two-dimensional steady non-isentropic analysis. Anti-shock criterion, and shock tube simulations for isentropic flows. European Journal of Mechanics B/Fluids 8(4):307-326, 1989.

[15] J. P. Petit, B. Lebrun. Theoretical analysis of shock wave anihilation with MHD force field. In 11th International Conference on MHD Electrical Power Generation. Proceedings III, part 9 - Fluid dynamics, pp. 748-753. Beijing, China, 1992. Article 4.
[16] J. P. Petit, J. Valensi. Growth rate of electrothermal instability and critical Hall parameter in closed-cycle MHD generators when the electron mobility is variable. CRAS, Academy of Science 269, 1969.

[17] J. P. Petit, J. Valensi, J. P. Caressa. Electrical characteristics of a converter using as a conversion fluid a binary mix of rare gases with non-equilibrium ionization. In 8th International Conference on MHD Electrical Power Generation, proceedings 3. International Atomic Energy Agency, Varsovie, Pologne, 1968.

[18] J. P. Petit, J. Valensi, J. P. Caressa. Theoretical and experimental study in shock tube of non-equilibrium phenomena in a closed-cycle MHD generator. In 8th International Conference on MHD Electrical Power Generation, proceedings 2, pp. 745-750. International Atomic Energy Agency, Varsovie, Poland, 1968.

[19] J. P. Petit, M. Viton. Convertisseurs MHD d'un genre nouveau. Appareils a induction (New MHD converters: induction machines). CRAS, French Academy of Sciences 284:167-179, 1977.

[20] E. P. Velikhov. Hall instability of current-carrying clightly-ionized plasmas. In 1st International Symposium on Magnetoplasmadynamics Electrical Power Generation. Newcastle-upon-Tyne, England, 1962. Paper 47.

[21] B. Zauderer. Experimental study of non equilibrium ionzation in MHD generators. AIAA Jr 4(6):701-707, 1968. 